\title{
La falacia de la validación personal: Más efectos placebo
}

\section{M. ${ }^{a}$ Dolores Avia, Javier Senén \\ y Alberto Simón \\ Universidad Complutense}

\section{INTRODUCCION}

Teóricamente, la utilización masiva de las baterías de tests responde a la confianza que los profesionales del diagnóstico ponen en ellas, en especial en su fiabilidad y validez. Pero quizá también la aceptación de que disfrutan entre muchos psicólogos se deba a la confianza que las tiene el propio sujeto que las recibe, que muchas veces, y aun sin ser cierta, se rinde a la «evidencia» que le presenta el resultado de un test.

Parece haber quedado claro (Forer, 1949; Snyder y Larson, 1972; Avia y Bragado, 1975; Collins, Dmitruk y Ranneuy, 1977; Labrador, 1980), que las personas aceptan de muy buen grado, como ciertos, informes falsos de personalidad, supuestamente obtenidos de ellos mediante tests. De este fenómeno se han propuesto diversas explicaciones: el prestigio del experimentador, el carácter mágico que para algunos sujetos tienen las pruebas, la aquiescencia, el efecto de la deseabilidad social, el carácter reforzante de un informe personal, etc.

El efecto del informe, se pensó en un principio, podía ser dependiente del prestigio con que el sujeto rodea al experimentador que lo elabora, especialmente cuando éste es una figura importante para él, como un profesor o terapeuta. Sin embargo, Ulrich, Stachnik y Stainton (1963) y Snyder y Larson (1972), probaron que el efecto era independiente del prestigio, al aceptarse igualmente los informes elaborados por compañeros de clase. Es probable, entonces, que sea el propio instrumento de medida el que se vea investido de ciertas cualidades que garanticen la veracidad de sus conclusiones. Es significativo que la aceptación del informe ocurre independientemente del tipo de prueba utilizada para elaborar el diagnóstico. Michels y Layne (1980) no encontraron ningún efecto debido a utilizar como prueba el MMPI, Rorschach, entrevistas estructuradas o entrevistas no estructuradas; Collins, Dmitruk y Ronney (1977) no obtuvieron diferencias al utilizar el MAS y un humorístico «test cerebral de la hipótesis nula», y lo que es más, los estudiantes aceptaron también como suyos informes elaborados por el psicólogo sin haberles pasado previamente ninguna prueba (la confianza en la intuición del psicólogo es, a todas luces, admirable).

La deseabilidad social del informe parece determinar de alguna forma su aceptación, si bien hay problemas al conceptuar esta variable. Aunque en general los informes favorables se aceptan más unánimemente que los desfavorables (Collins y otros, 1977; Greene, Harris y Macon, 1979; Michels y Lay- 
ne, 1980; Layne y Ally, 1980), queda por dilucidar si lo que se considera favorable es lo más deseable socialmente, o más simplemente, aquello que es común en un grupo y que por ello tiende a aceptarse mejor (ver al respecto Collins y otros, op. cit., y Weinberger y Bradley, 1980). Otras explicaciones han considerado la aquiescencia al sesgo de respuesta que determina en mayor medida la aceptación, especialmente si la redacción del informe utiliza afirmaciones vagas, ambiguas, muy generales y con un lenguaje cientifista. Parece determinante también el efecto reforzante que podría suponer recibir un informe especialmente elaborado para uno. Aunque los informes que describen a «un estudiante típicamente medio» se aceptan mejor de lo que cabría esperar por azar (Collins y otros, 1977; Snyder y Larson, 1972), esta aceptación es menor que cuando está elaborada para uno; y la misma información se acepta en mayor medida cuando se presenta en forma de informe (resultado de unas pruebas) que en forma de un cuestionario más que el sujeto debe valorar (Michels y Layne, 1980; Layne y Ally, 1980). En todo caso, es probable que sea más de un factor el que determine la paradójica aceptación de afirmaciones muchas veces opuestas a lo que el sujeto ha dicho de sí mismo en los cuestionarios que ha rellenado.

Se han verificado, por otra parte, ciertas relaciones entre la aceptación del informe y características de personalidad. Avia y Bragado (1975) encontraron que las mujeres más flexibles (y, en contraste, los varones más rígidos) y con mayor ansiedad estimulante del rendimiento, tendían a aceptarlo mejor. Labrador halló algunas relaciones curvilíneas entre neuroticismo y aceptación, siendo el grupo neurótico introvertido el de mayor aceptación del informe. Por lo demás, ni el sexo ni el nivel intelectual aparecen relacionados con este fenómeno.

La mayoría de las investigaciones sobre el tema se ha realizado con muestras de estudiantes de Psicología. Uno de los objetivos del presente trabajo es el de ampliar la muestra a otros estudiantes, concretamente a dos grupos tan supuestamente diferentes como los de Ciencias Políticas y los de una ingeniería técnica. Nos proponemos también estudiar el papel que tiene el contenido del informe en su aceptación. Por ello, y con modificaciones menores, hemos querido comparar el informe originalmente elaborado por Avia y Bragado, posteriormente utilizado con revisiones por Labrador, con una forma que conservaba la misma formulación y redacción, pero de contenido opuesto. Finalmente, y con el fin de controlar la deseabilidad social, hemos incluido entre los informes de personalidad una medida de esta variable. Al realizar el trabajo, hemos partido de las siguientes hipótesis:

1. Esperamos obtener un alto grado de acuerdo, superior al punto medio de la escala de evaluación, en las dos formas del informe, y una aceptación mayor en la forma originalmente elaborada que en la opuesta.

2. Esperamos obtener un alto grado de aceptación, superior al punto medio de la escala, en las dos muestras de estudiantes, pero menor en la de estudiantes de Ciencias Políticas.

3. Esperamos encontrar una correlación positiva entre las puntuaciones en deseabilidad social y la aceptación del informe.

\section{METODO}

\subsection{Sujetos y procedimiento}

Participaron en este estudio 40 sujetos ( 8 mujeres y 32 varones) de edades comprendidas entre diecinueve y veintitrés años, alumnos de la Univer- 
sidad Complutense de Madrid. La muestra estaba integrada por 16 alumnos (7 mujeres y 9 varones) de segundo curso de la Facultad de Ciencias Políticas y Sociología, y por 24 (23 varones y una mujer) de la Escuela Técnica Superior de Ingenieros de Telecomunicación ${ }^{1}$.

Los profesores de ambos grupos les informaron que en la Facultad de Psicología se estaba llevando a cabo una investigación con vistas a mejorar los instrumentos de evaluación de la personalidad, para lo cual se requería su colaboración, que consistía en rellenar unos cuestionarios. Tras ello, y para agradecer su ayuda, los profesores de Psicología les entregarían un informe personal y secreto con los resultados obtenidos.

En una primera sesión se aplicaron las siguientes pruebas de personalidad: Cuestionario EN-36 de Brengelman (versión española de V. Pelechano), que aísla los factores de introversión social y neuroticismo; cuestionario AP de rigidez de Fish (adaptación de V. Pelechano), que mide los factores de rigidez de principios y rigidez laboral; cuestionario DR de depresión situacional, adaptación de V. Pelechano del de Von Zersen, Keller y Rey, y cuestionario DS de deseabilidad social de Marlowe y Crowne, en el que se han distinguido dos factores: la tendencia a aceptar items favorables (DS+) y la tendencia a rechazar items desfavorables (DS-).

Terminada la aplicación de las pruebas, se prometieron los resultados para una segunda sesión, que debido a las vacaciones de Navidad se llevó a cabo aproximadamente mes y medio después de la primera.

Para esta segunda sesión, se elaboraron dos informes diferentes, tomando como base el de Avia y Bragado (1975), y utilizando únicamente los ocho items de aquél que se correspondían con las pruebas que se habían utilizado. Uno de los informes ( $f$ forma $B$ ») era el mismo utilizado en aquella ocasión ${ }^{2}$. La forma A se elaboró como un «negativo» de la B, conservando la construcción gramatical y variando únicamente el contenido (así, si en una forma se dice «ligeramente introvertido», en la otra, «ligeramente extrovertido»; véase apéndice). La mitad de los sujetos en cada grupo recibió la forma $\mathrm{A}$ y la otra mitad la B. Encima de cada informe aparecía el nombre y apellidos del alumno. Se pidió a los sujetos que leyesen los resultados con detenimiento y calificasen en una escala de 1 a 7 su grado de acuerdo con cada enunciado. Una vez entregadas las evaluaciones, se explicó el desarrollo del experimento y se discutieron sus implicaciones para el diagnóstico.

\section{RESULTADOS}

Comparación entre las medias totales de las dos muestras

Las puntuaciones medias, así como las desviaciones típicas obtenidas por los grupos de Sociología y Telecomunicaciones en el informe global, aparecen en la Tabla 1. Hemos considerado inicialmente la totalidad de la muestra, independientemente de que se hubiera recibido la forma $\mathrm{A} \circ \mathrm{B}$.

El análisis estadístico aplicado - prueba de $\mathrm{T}$ - no permite apreciar, con $p<001$, diferencias significativas entre ambos grupos $\left(t_{38}=0,16\right)$. Es decir, las dos muestras aceptaron en la misma medida el informe, en la dirección propuesta por nuestra hipótesis segunda (las calificaciones sobrepasarían la puntuación media de la escala), aunque, frente a lo que indicábamos en esta misma hipótesis, no hubo diferencias entre ellas. Esto hace suponer que el fenómeno de la aceptación es más general de lo que en principio pensábamos. 
Medias y desviaciones tipicas (entre paréntesis) de las dos muestras en las dos formas del informe $y$ en total.

\begin{tabular}{lccc}
\hline & Total & $A$ & $B$ \\
\hline Sociología & 5,52 & 5,35 & 5,62 \\
& $(1,55)$ & $(1,75)$ & $(1,37)$ \\
Telecomunicación & 5,27 & 5,37 & 5,15 \\
& $(1,56)$ & $(1,50)$ & $(1,44)$ \\
\hline
\end{tabular}

Comparación entre las dos formas (A y B) del informe

Los resultados fueron análogos a los del apartado anterior. No se encontraron diferencias significativas entre la puntuación media del informe $A$ y la del $B$, ni en Sociología $\left(t_{18}=0,32\right)$ ni en Telecomunicación $\left(t{ }_{22}=0,35\right)$, y en ambos casos fueron superiores al punto medio de la escala. Es decir, la aceptación global del informe fue independiente del contenido del mismo.

\section{Comparación entre cada ítem y su opuesto (formas A y B)}

A pesar de la no existencia de diferencias significativas entre las medias totales de las dos formas del informe, quisimos buscar las diferencias entre ambas en cada uno de los ocho items. Comparamos, pues, cada uno de los ocho enunciados de la forma $A$ con el correspondiente de la forma B. Los resultados aparecen en las Tablas 2 y 3 .

En Políticas se dieron diferencias significativas entre las formas $A$ y $B$ en los items 3, 4 y 5 . Los dos primeros se referían a dos aspectos de rigidez-flexibilidad. El ítem 4, que en la forma $A$ obtuvo la puntuación media más alta en todos los casos $(6,87)$, presentaba al sujeto como flexible y condescendien-

\section{TABLA 2}

Puntuaciones medias de los ocho items del informe.

Items

\begin{tabular}{|c|c|c|c|c|c|c|c|c|c|}
\hline & & 1 & 2 & 3 & 4 & 5 & 6 & 7 & 8 \\
\hline \multirow{2}{*}{ 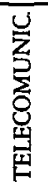 } & F. A & $\begin{array}{c}5,12 \\
(1,76)\end{array}$ & $\begin{array}{l}5,25 \\
(1,3)\end{array}$ & $\begin{array}{l}6,5 \\
\text { (1) }\end{array}$ & $\begin{array}{c}6,87 \\
(0,41)\end{array}$ & $\begin{array}{c}3,75 \\
(2,38)\end{array}$ & $\begin{array}{c}5,37 \\
(1,22)\end{array}$ & $\begin{array}{c}6,12 \\
(0,6)\end{array}$ & $\begin{array}{c}4,87 \\
(4,04)\end{array}$ \\
\hline & F. B & $\begin{array}{c}5,87 \\
(1,27)\end{array}$ & $\begin{array}{c}6 \\
(1,32)\end{array}$ & $\begin{array}{c}4,5 \\
(1,22)\end{array}$ & $\begin{array}{c}4,12 \\
(1,54)\end{array}$ & $\begin{array}{c}6,12 \\
(0,78)\end{array}$ & $\begin{array}{c}5,37 \\
(1,22)\end{array}$ & $\begin{array}{c}6,25 \\
(0,97)\end{array}$ & $\begin{array}{c}6,25 \\
(0,66)\end{array}$ \\
\hline \multirow{2}{*}{ 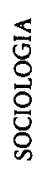 } & F. $A$ & $\begin{array}{c}4,77 \\
(1,53)\end{array}$ & $\begin{array}{c}4,23 \\
(1,67)\end{array}$ & $\begin{array}{c}5,54 \\
(1,21)\end{array}$ & $\begin{array}{c}5,85 \\
(1,28)\end{array}$ & $\begin{array}{c}5,31 \\
(1,72)\end{array}$ & $\begin{array}{c}5,69 \\
(1,07)\end{array}$ & $\begin{array}{c}4,85 \\
(1,75)\end{array}$ & $\begin{array}{c}5,23 \\
(2,08)\end{array}$ \\
\hline & F. B & $\begin{array}{c}5,36 \\
(0,77)\end{array}$ & $\begin{array}{c}5,91 \\
(1,44)\end{array}$ & $\begin{array}{c}4,64 \\
(1,35)\end{array}$ & $\begin{array}{c}5,09 \\
(0,99)\end{array}$ & $\begin{array}{c}5,18 \\
(1,59)\end{array}$ & $\begin{array}{c}4,09 \\
(2,02)\end{array}$ & $\begin{array}{c}6,18 \\
(0,94)\end{array}$ & $\begin{array}{c}6 \\
(1,13)\end{array}$ \\
\hline
\end{tabular}

Las desviaciones típicas aparecen entre paréntesis. 
Valores de «t» para las diferencias entre cada uno de los ocho items en las dos formas del informe.

Items del informe

\begin{tabular}{ccccccccc}
\hline & 1 & 2 & 3 & 4 & 5 & 6 & 7 & 8 \\
\hline POLIT. & & 1,06 & $4,76 *$ & $4,51 *$ & $2,04 *$ & 0 & 0,30 & 0,88 \\
TELEC. & 0,89 & $2,47 *$ & 1,67 & 0,32 & 0,18 & $2,35 *$ & $2,15 *$ & 0,24 \\
& 1,11 & & & & & & & \\
\hline
\end{tabular}

Con asterisco, los valores significativos.

te en el trabajo «aunque lo valorase». La forma opuesta (individuo exigente y no condescendiente en el trabajo) fue significativamente menos aceptada. Estos datos presentan una curiosa contradicción con los resultados del ítem 3 , en el que los alumnos de Políticas se muestran más de acuerdo $(\overline{\mathrm{X}}=6,5)$ con una consideración inflexible de sí mismos «para quienes los grandes principios como justicia y libertad ocupan un lugar más importante que consideraciones personales». La afirmación opuesta, más bien flexible, fue significativamente menos aceptada $(\overline{\mathrm{X}}=4,5)$.

Asimismo, los alumnos de Políticas aceptaron significativamente mejor la versión que les presentaba como inconformistas, y con criterios demasiado exigentes en la selección de sus amigos (ítem 5). Ninguna de estas diferencias aparecieron en los alumnos de Telecomunicación, que, sin embargo, aceptaron de modo diferente las dos formas de los items 2, 6 y 7: prefirieron que les dijeran introvertidos (ítem 2), que «no tenían un sentido excesivamente crítico ante situaciones sociales y tenían una visión más bien optimista de la sociedad española» (ítem 6) y que «eran capaces de entender los actos de los demás, aun sin compartir sus intereses». A su vez, ninguna de estas diferencias se dio en Políticas.

Parece, pues, que mientras que los alumnos de Políticas «prefieren» verse como flexibles en el trabajo, inflexibles en los grandes principios sociales, e inconformistas y críticos en las relaciones personales, los de Telecomunicación gustan de verse como introvertidos, no demasiado críticos sobre situaciones sociales, optimistas con el medio social y comprensivos con los demás.

\section{Relaciones entre aceptación del informe y variables de personalidad}

En la Tabla 4 se muestran las correlaciones de Pearson entre la aceptación de las dos formas del informe y las puntuaciones en los cuestionarios de personalidad. Las correlaciones se han calculado conjuntamente en las dos muestras de sujetos. Ninguna de las correlaciones con personalidad ni con el sexo alcanzó significación estadística. Destaca, en cualquier caso, la tendencia negativa de las correlaciones entre aceptación y las tres medidas de deseabilidad social, contra lo que cabía esperar según nuestra hipótesis 3 .

En la Tabla 5 aparecen las medias y desviaciones típicas de las dos muestras en las variables de personalidad. Como se ve por las pruebas de $t$, los alumnos de Políticas presentan puntuaciones significativamente más altas en 


\begin{tabular}{clcc}
\hline & & \multicolumn{2}{c}{ Aceptación informe } \\
\cline { 3 - 4 } & Extraversión & $\begin{array}{c}\text { Forma A } \\
(\mathbf{n}=21)\end{array}$ & $\begin{array}{c}\text { Forma B } \\
(\mathbf{n}=19)\end{array}$ \\
\cline { 3 - 4 } & Neuroticismo & $-0,10$ & $-0,02$ \\
& Des. social (total) & 0,03 & 0,25 \\
Variables & Des. social (+) & $-0,31$ & $-0,27$ \\
de & Des. social (-) & $-0,24$ & $-0,16$ \\
personalidad & Rigidez princip. & $-0,31$ & $-0,33$ \\
& Rigidez laboral & 0,13 & $-0,10$ \\
& Depresión situac. & 0,10 & $-0,06$ \\
& Sexo & $-0,07$ & 0,41 \\
& & 0,07 & 0,11 \\
\hline
\end{tabular}

$\mathrm{p}<0,05=0,43(\mathrm{n}: 21) ; 0,45$ (n: 19)

$\mathrm{p}<0,01=0,55$ (n: 21);0,57 (n: 19)

TABLA 5

Resultados reales en las variables de personalidad. Medias, desviaciones tipicas $y$ pruebas de $\mathrm{t}$ en las dos muestras de alumnos.

\begin{tabular}{lcrll}
\hline & \multicolumn{1}{c}{$\begin{array}{c}\text { Políticas } \\
(\mathbf{n}=20)\end{array}$} & $\begin{array}{c}\text { Telecom. } \\
(\mathbf{n}=40)\end{array}$ & \multicolumn{1}{c}{$t$} \\
\cline { 2 - 5 } Extrav. & $4,95(3,18)$ & $5,65(3,23)$ & $0,81 \quad(\mathrm{n}, 5)$ \\
Neurot. & $13,35(5,14)$ & $9,70(5,62)$ & $2,48^{*}$ \\
Rigidez principios & $11,20(4,34)$ & $15,20(6,40)$ & $2,56^{*}$ \\
Rigidez laboral & $5,50(2,18)$ & $6,87(2,98)$ & $1,86 \quad(\mathrm{n} .5)$ \\
Depres. situac. & $8,50(6,75)$ & $5,75(6,86)$ & 1,5 & $(\mathrm{n} .5)$ \\
Deseab. social (+) & $9,85(2,71)$ & $11,15(3,07)$ & 1,63 (n.5) \\
Deseab. social (-) & $6,30(2,99)$ & $7,30(3,02)$ & $1,23 \quad(\mathrm{n} .5)$ \\
\hline
\end{tabular}

* Hemos analizado aquí los cuestionarios de todos los sujetos, aunque ya hemos indicado que algunos no recogieron el informe y no constan sus datos en los análisis anteriores.

Neuroticismo que los de Telecomunicación, siendo éstos, en cambio, más rígidos que aquéllos en lo referente a principios. Estas diferencias chocan claramente con los resultados de la aceptación del informe: aun siendo más inflexibles, los alumnos de Telecomunicación no aceptan mejor el informe que los presenta como tales, mientras que sí lo hacen los alumnos de Políticas que, sin embargo, y comparativamente, son más flexibles.

\section{DISCUSION}

Los resultados de este trabajo sugieren que la aceptación del diagnóstico que ofrece un profesional es un fenómeno general independiente de las características personales de los individuos que lo reciben. Tomados globalmente, ambos informes se aceptaron igualmente bien y de modo similar en las 
dos muestras de sujetos, lo cual, como otros trabajos anteriores, pone en entredicho el fenómeno de la validación personal con que a menudo gustan de apoyar su trabajo los profesionales de la terapia y el diagnóstico. La confirmación por el sujeto de los juicios que de él hacen los demás en modo alguno constituye siempre una verdadera validación.

Sobre este efecto general, sin embargo, se superponen interesantes efectos específicos debidos al contenido concreto de algunas afirmaciones, que interactúa con las características profesionales de los sujetos. Parece, pues, que independientemente de sus puntuaciones reales en las pruebas, los estudiantes de nuestro trabajo difieren en la imagen de personalidad que más dispuestos están a aceptar sobre sí mismos. Por lo que respecta al grupo de ingeniería, esta imagen es consistente con un estereotipo social más bien «adaptado» (extravertido, comprensivo con los demás, optimista sobre la sociedad, no muy crítico), con el que a veces se les identifica. La imagen preferida por los alumnos de Sociología apunta, en cambio, al inconformismo, la defensa de ciertos principios y la flexibilidad en el trabajo.

Las características de nuestro estudio no permiten dilucidar el papel que la deseabilidad social ha cumplido en estos resultados. En la elaboración del informe inicial (forma B) creímos dibujar la imagen que el estudiante estaría más dispuesto a aceptar de sí mismo; sin embargo, en muchos casos ha sido más aceptada la imagen opuesta. Por tanto, y frente a nuestras suposiciones, no hubo ninguna forma globalmente más aceptada que la otra. Esto podría explicar, quizá, la falta de correlación entre deseabilidad social y aceptación global del informe. Por otro lado, tampoco evaluamos independientemente la deseabilidad social de cada enunciado.

Una limitación importante del trabajo fue el tamaño reducido de la muestra, limitación que se acrecentó con la mortandad experimental: de 76 sujetos que inicialmente rellenaron las pruebas, sólo se incluyeron en el estudio algo más de la mitad. El análisis de esta mortandad ofrece, sin embargo, cierto interés. En la Facultad de Ciencias Políticas y Sociología se perdieron diez sujetos, y dieciséis en la E. T.S. de Ingenieros de Telecomunicación, en la mayor parte por no haber acudido a clase el día en que se repartió el informe, excepto algunos sujetos que no rellenaron bien los cuestionarios. Los restantes dieciséis sujetos pertenecían al segundo curso de la Facultad de Psicología ${ }^{3}$ y no se incluyen en el estudio porque, excepto tres de ellos, no acudieron a recibir el informe. Evidentemente, son muchas las razones que podrían explicar este hecho, pero una de ellas podría ser una falta de interés real por obtener su «informe» de personalidad. Quizá los estudiantes de Psicología estén en este momento ${ }^{4}$ más saturados de pruebas psicológicas que los alumnos de otras especialidades, o quizá confíen menos en ellas. En cualquier caso, la Tabla 6 expresa las puntuaciones reales de dichos sujetos en las pruebas que se les aplicaron. En relación con los otros sujetos, los de psicología aparecen como más extravertidos que los de políticas $(\mathrm{t}=2,01, \mathrm{p}<0,05)$ y, con mayores puntuaciones en neuroticismo $(t=2.84, \mathrm{p}<0.1)$ y despresión situacional $(\mathrm{t}=2.24, \mathrm{p}<0,01)$ que los de Telecomunicación, a la vez que puntúan menos en los dos factores de deseabilidad social $(t=2.24$ y $3.12, p<.01$ respectivamente). En suma, destaca en ellos el carácter no muy «deseable» de su perfil, que difiere sensiblemente del de Telecomunicaciones y se acerca más al de Políticas. A título meramente indicativo, la comparación visual con el perfil de los estudiantes de psicología de hace casi diez años (según Avia-Bragado, 1975) subraya el carácter «negativo» de esta muestra (las diferencias son altamente significativas en las variables de extraversión $(t=3.7, p<0,001)$ y neuroticismo $(t=4.5, p<0,001)$, lo cual quizá tenga cierta relación con lo ocurrido en nuestro trabajo. 
Medias y $D$. T en las variables de personalidad de los estudiantes de Psicología. A la derecha, los resultados del estudio anterior de Avia y Bragado (1975).

\begin{tabular}{lcc}
\hline & $\begin{array}{c}\text { Psicología 1982 } \\
(\mathrm{n}=16)\end{array}$ & $\begin{array}{c}\text { Psicología 1975 } \\
(\mathrm{n}=75)\end{array}$ \\
\cline { 2 - 3 } Extrav. & $7,12(3,20)$ & $4,16(2,97)$ \\
Neurot. & $14,06(4,59)$ & $7,74(5,38)$ \\
Rigidez principios & $13,43(4,48)$ & $14,05(6,73)$ \\
Rigidez laboral & $7,06(3,29)$ & $6,24(2,92)$ \\
Despres. situacional & $10,00(5,91)$ & $9,63(7,73)$ \\
Deseab. social (+) & $8,37(3,18)$ & - \\
Deseab. social (-) & $5,31(2,41)$ & - \\
\hline
\end{tabular}

Queremos rebatir, para terminar, la tesis, esbozada en algunos trabajos, de que hay ciertos juicios «universalmente válidos» y que por tanto son difíciles de rechazar. Baucom y Greene (1979) han demostrado claramente que estos juicios no se aceptan universalmente si no se presentan en forma de resultados de personalidad, y que además los sujetos pueden predecir con gran precisión el porcentaje de personas que los van a aceptar. Todo hace pensar, en suma, que es el hecho de entregar un informe personal, resultado de unas pruebas psicológicas, lo que activa esta curiosa y general aceptación. Quién es la persona que dé el informe y cuál el tipo de pruebas usadas, parece poco importante. El contenido del informe, en cambio, parece afectar diferencialmente a grupos profesionales diferentes.

\section{Referencias}

Avia, M. D., y Bragado, C.: «Los informes de personalidad y la credibilidad ante los tests: un efecto placebo». Análisis y Modificación de Conducta, 1975, 1, 33-54.

BAuCOM, D. H., y GREENE, R. L.: «The universality of generalized personality statements». Journal of Personality Assessment, 1979, 43, 5, 497-500.

Coluins, R. W.; DMitruk, V. M., y Ranney, J. T.: «Personal validation: some empirical and ethical considerations». Journal of Consulting and Clinical Psychology, 1977, 45(1), 70-77.

FORER, B. R.: «The fallacy of personal validation: a classroom demostration of gullibillity». Journal of Abnormal and Social Psychology, 1949, 44, 118-123.

Greene, R. L.; HARRIS, M. E., y MACON, S. R.: «Another look at personal validation». Journal of Personality Assessment, 1979, 43, 4, 419-423.

LABRADOR, F. J.: «Susceptibilidad a la sugestión en función de los factores de personalidad». Estudios de Sicologia, 1980, 3, 15-27.

LAYNE, C., y ALLY, C.: «How and why people accept personality feedback». Joumal of Assessment, 1980, 44, S, 541-546.

MICHELS, P. J., y LAYNE, C.: «Inventory responding models people's acceptance of feedback derived from tests and from interviewsm. Joumal of Personality Assessment, 1980, 44, 3, 302-306.

SNYDER, C. R., y LARSON, G. R.: «A further look at student acceptance of general personality interpretations». Journal of Consulting and Clinical Psychology, 1972, 38, 384-388.

SNYDER, C. R.; SHENKEL, R. J., y LOWERY, C. R.: «Acceptance of personality interpretations: the "Barnum effect" and beyond». Journal of Consulting and Clinical Psychology, 1977, 45, 104-114.

UlRich, R. E., STACHNIK, J. J. y Stainton, S. R.: «Student acceptance of generalized personality interpretations». Psychological Reports, 1963, 13, 831-834.

WEINBERGER, L. J., y BRADLEY, L. A.: «Effects of favorability and type of assessment device upon acceptance of general personality interpretations». Joumal of Personality Assessment, 1980, 44, 1, 44-47.

\section{Notas}

1 Agradecemos a los profesores E. Crespo y S. Lorente la participación prestada.

2 Los items 1, 2, 5, 6 y 7 tenían idéntica formulación, y los números 3,4 y 8 recibieron modificaciones menores que aludian a las diferentes circunstancias de aplicación.

3 Agradecemos su colaboración al profesor J. F. Labrador.

${ }_{4}$ Queremos llamar la atención al hecho de que, por razones ajenas a los autores, hay una importante distancia temporal entre la fecha de entrega de este trabajo y la fecha de su publicación. 